Computer Science \& Engineering: An International Journal (CSEIJ), Vol.1, No.5, December 2011

\title{
Global ChaOs SyNCHRONIZATION OF Hyperchaotic Pang and Hyperchaotic WaNg SySTEMS VIA ADAPTIVE CONTROL
}

\author{
Sundarapandian Vaidyanathan $^{1}$ and Karthikeyan Rajagopal ${ }^{2}$ \\ ${ }^{1}$ Research and Development Centre, Vel Tech Dr. RR \& Dr. SR Technical University \\ Avadi, Chennai-600 062, Tamil Nadu, INDIA \\ sundarvtu@gmail. com \\ ${ }^{2}$ School of Electronics and Electrical Engineering, Singhania University \\ Dist. Jhunjhunu, Rajasthan-333 515, INDIA \\ rkarthiekeyanegmail.com
}

\begin{abstract}
This paper investigates the global chaos synchronization of identical hyperchaotic Wang systems, identical hyperchaotic Pang systems, and non-identical hyperchaotic Wang and hyperchaotic Pang systems via adaptive control method. Hyperchaotic Pang system (Pang and Liu, 2011) and hyperchaotic Wang system (Wang and Liu, 2006) are recently discovered hyperchaotic systems. Adaptive control method is deployed in this paper for the general case when the system parameters are unknown. Sufficient conditions for global chaos synchronization of identical hyperchaotic Pang systems, identical hyperchaotic Wang systems and non-identical hyperchaotic Pang and Wang systems are derived via adaptive control theory and Lyapunov stability theory. Since the Lyapunov exponents are not required for these calculations, the adaptive control method is very convenient for the global chaos synchronization of the hyperchaotic systems discussed in this paper. Numerical simulations are presented to validate and demonstrate the effectiveness of the proposed synchronization schemes.
\end{abstract}

\section{KEYWORDS}

Adaptive Control, Hyperchaos, Synchronization, Hyperchaotic Pang System, Hyperchaotic Wang System.

\section{INTRODUCTION}

Chaotic systems are dynamical systems that are highly sensitive to initial conditions. The sensitive nature of chaotic systems is commonly called as the butterfly effect [1]. Since chaos phenomenon in weather models was first observed by Lorenz in 1963 [2], a large number of chaos phenomena and chaos behaviour have been discovered in physical, social, economical, biological and electrical systems.

A hyperchaotic system is usually characterized as a chaotic system with more than one positive Lyapunov exponent implying that the dynamics expand in more than one direction giving rise to "thicker" and "more complex" chaotic dynamics. The first hyperchaotic system was discovered by Rössler in 1979 [3].

DOI : 10.5121/cseij.2011.1502 
Chaos is an interesting nonlinear phenomenon and has been extensively studied in the last two decades [1-40].

Synchronization of chaotic systems is a phenomenon which may occur when two or more chaotic oscillators are coupled or when a chaotic oscillator drives another chaotic oscillator. Because of the butterfly effect which causes the exponential divergence of the trajectories of two identical chaotic systems started with nearly the same initial conditions, synchronizing two chaotic systems is seemingly a very challenging problem.

In 1990, Pecora and Carroll [4] deployed control techniques to synchronize two identical chaotic systems and showed that it was possible for some chaotic systems to be completely synchronized. From then on, chaos synchronization has been widely explored in a variety of fields including physical systems [5], chemical systems [6], ecological systems [7], secure communications [810], etc.

In most of the chaos synchronization approaches, the master-slave or drive-response formalism is used. If a particular chaotic system is called the master or drive system and another chaotic system is called the slave or response system, then the idea of the synchronization is to use the output of the master system to control the slave system so that the output of the slave system tracks the output of the master system asymptotically.

Since the seminal work by Pecora and Carroll [4], a variety of impressive approaches have been proposed for the synchronization of chaotic systems such as the OGY method [11], active control method [12-16], adaptive control method [17-22], sampled-data feedback synchronization method [23], time-delay feedback method [24], backstepping method [25-26], sliding mode control method [27-32], etc.

In this paper, we investigate the global chaos synchronization of uncertain hyperchaotic systems, viz. identical hyperchaotic Pang systems ([33], 2011), identical hyperchaotic Wang systems ([34], 2006) and non-identical hyperchaotic Pang and hyperchaotic Wang systems. We consider the general case when the parameters of the hyperchaotic systems are unknown.

This paper is organized as follows. In Section 2, we provide a description of the hyperchaotic systems addressed in this paper, viz. hyperchaotic Pang system (2011) and hyperchaotic Wang system (2006). In Section 3, we discuss the adaptive synchronization of identical hyperchaotic Pang systems. In Section 4, we discuss the adaptive synchronization of identical hyperchaotic Wang systems. In Section 5, we discuss the adaptive synchronization of non-identical hyperchaotic Pang and hyperchaotic Wang systems. In Section 6, we summarize the main results obtained in this paper.

\section{SYSTEMS DESCRIPTION}

The hyperchaotic Pang system ([33], 2011) is described by the dynamics

$$
\begin{aligned}
& \dot{x}_{1}=a\left(x_{2}-x_{1}\right) \\
& \dot{x}_{2}=c x_{2}-x_{1} x_{3}+x_{4} \\
& \dot{x}_{3}=-b x_{3}+x_{1} x_{2} \\
& \dot{x}_{4}=-d\left(x_{1}+x_{2}\right)
\end{aligned}
$$

where $x_{1}, x_{2}, x_{3}, x_{4}$ are the state variables and $a, b, c, d$ are positive, constant parameters of the system. 
Computer Science \& Engineering: An International Journal (CSEIJ), Vol.1, No.5, December 2011

The 4-D system (1) is hyperchaotic when the parameter values are taken as

$$
a=36, b=3, c=20 \text { and } d=2
$$

The state orbits of the hyperchaotic Pang chaotic system (1) are shown in Figure 1.
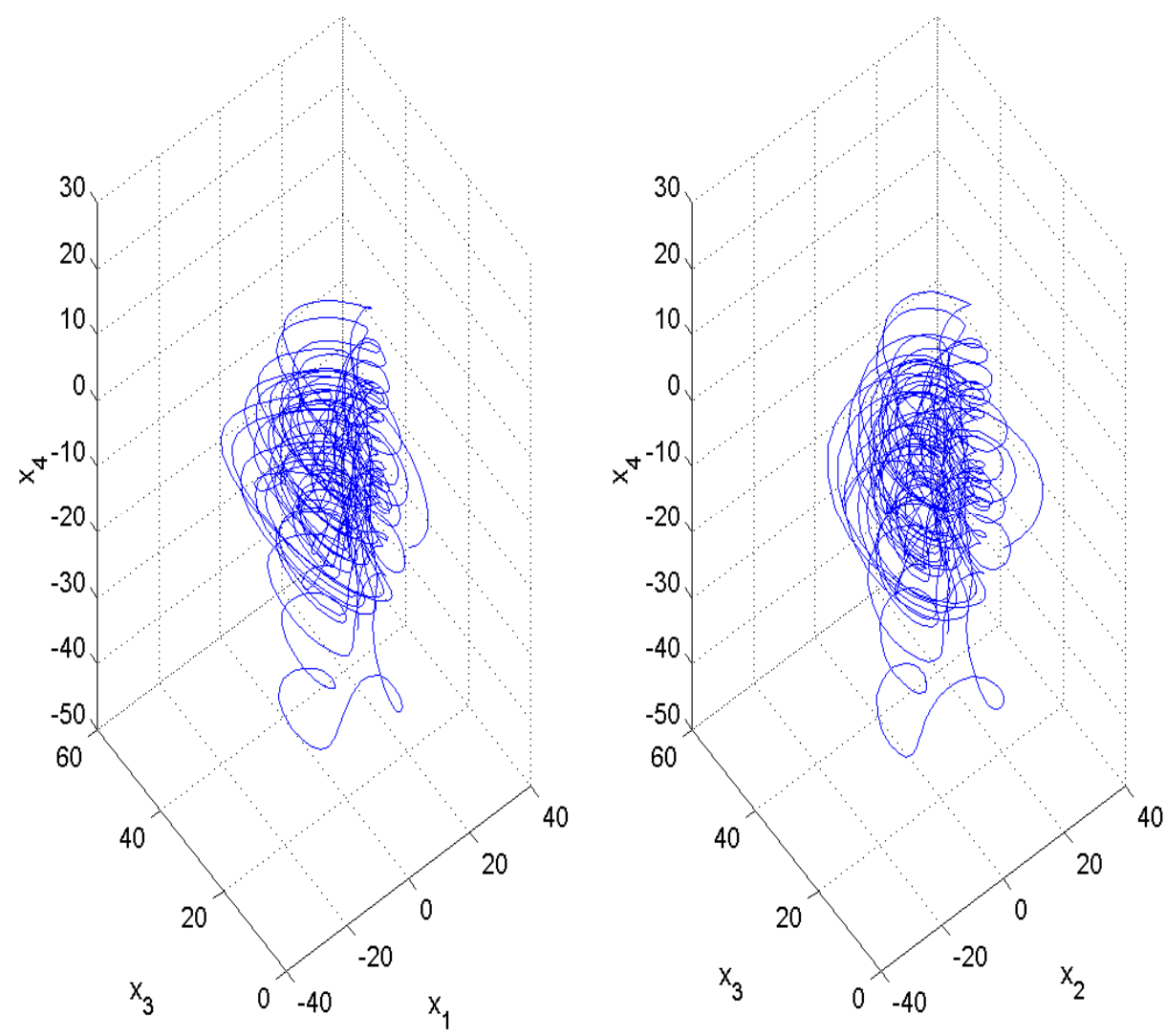

Figure 1. State Orbits of the Hyperchaotic Pang Chaotic System

The hyperchaotic Wang system ([34], 2006) is described by

$$
\begin{aligned}
& \dot{x}_{1}=\alpha\left(x_{2}-x_{1}\right) \\
& \dot{x}_{2}=\beta x_{1}-x_{1} x_{3}+x_{4} \\
& \dot{x}_{3}=-\gamma x_{3}+\varepsilon x_{1}^{2} \\
& \dot{x}_{4}=-\delta x_{1}
\end{aligned}
$$

where $x_{1}, x_{2}, x_{3}, x_{4}$ are the state variables and $\alpha, \beta, \gamma, \delta, \varepsilon$ are positive constant parameters of the system.

The 4-D system (2) is hyperchaotic when the parameter values are taken as 
Computer Science \& Engineering: An International Journal (CSEIJ), Vol.1, No.5, December 2011

$$
\alpha=10, \quad \beta=40, \quad \gamma=2.5, \quad \delta=10.6 \text { and } \varepsilon=4
$$

The state orbits of the hyperchaotic Wang chaotic system (2) are shown in Figure 2.
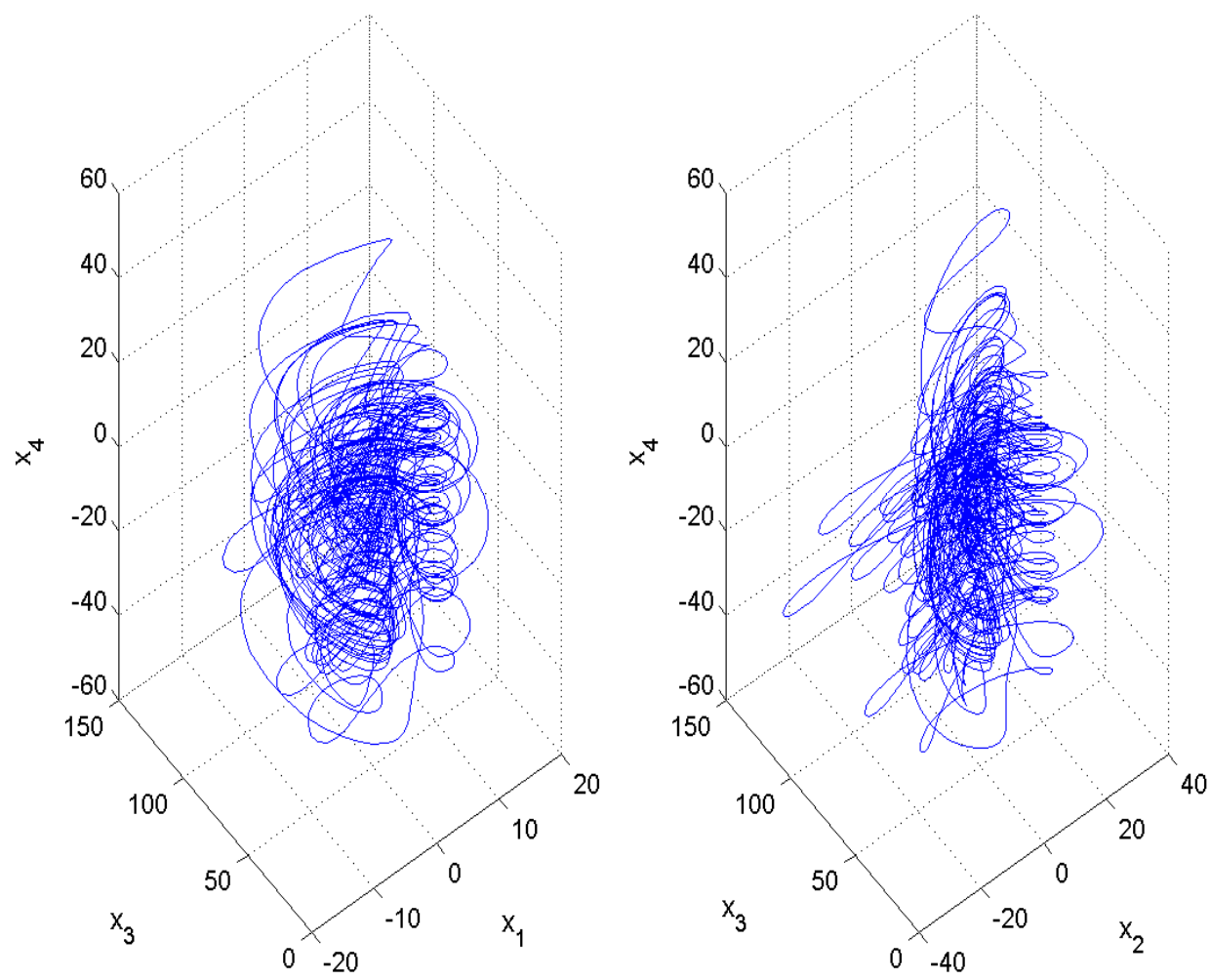

Figure 2. State Orbits of the Hyperchaotic Wang System

\section{Adaptive Synchronization of Identical Hyperchaotic Pang SYSTEMS}

\subsection{Theoretical Results}

In this section, we deploy adaptive control to achieve new results for the global chaos synchronization of identical hyperchaotic Pang systems ([33], 2011), where the parameters of the master and slave systems are unknown.

As the master system, we consider the hyperchaotic Pang dynamics described by

$$
\begin{aligned}
& \dot{x}_{1}=a\left(x_{2}-x_{1}\right) \\
& \dot{x}_{2}=c x_{2}-x_{1} x_{3}+x_{4} \\
& \dot{x}_{3}=-b x_{3}+x_{1} x_{2} \\
& \dot{x}_{4}=-d\left(x_{1}+x_{2}\right)
\end{aligned}
$$


Computer Science \& Engineering: An International Journal (CSEIJ), Vol.1, No.5, December 2011

where $x_{1}, x_{2}, x_{3}, x_{4}$ are the state variables and $a, b, c, d$ are unknown, real ,constant parameters of the system.

As the slave system, we consider the controlled hyperchaotic Pang dynamics described by

$$
\begin{aligned}
& \dot{y}_{1}=a\left(y_{2}-y_{1}\right)+u_{1} \\
& \dot{y}_{2}=c y_{2}-y_{1} y_{3}+y_{4}+u_{2} \\
& \dot{y}_{3}=-b y_{3}+y_{1} y_{2}+u_{3} \\
& \dot{y}_{4}=-d\left(y_{1}+y_{2}\right)+u_{4}
\end{aligned}
$$

where $y_{1}, y_{2}, y_{3}, y_{4}$ are the state variables and $u_{1}, u_{2}, u_{3}, u_{4}$ are the nonlinear controllers to be designed.

The chaos synchronization error is defined by

$$
e_{i}=y_{i}-x_{i}, \quad(i=1,2,3,4)
$$

The error dynamics is easily obtained as

$$
\begin{aligned}
& \dot{e}_{1}=a\left(e_{2}-e_{1}\right)+u_{1} \\
& \dot{e}_{2}=c e_{2}+e_{4}-y_{1} y_{3}+x_{1} x_{3}+u_{2} \\
& \dot{e}_{3}=-b e_{3}+y_{1} y_{2}-x_{1} x_{2}+u_{3} \\
& \dot{e}_{4}=-d\left(e_{1}+e_{2}\right)+u_{4}
\end{aligned}
$$

Let us now define the adaptive control functions

$$
\begin{aligned}
& u_{1}(t)=-\hat{a}\left(e_{2}-e_{1}\right)-k_{1} e_{1} \\
& u_{2}(t)=-\hat{c} e_{2}-e_{4}+y_{1} y_{3}-x_{1} x_{3}-k_{2} e_{2} \\
& u_{3}(t)=\hat{b} e_{3}+y_{1} y_{2}-x_{1} x_{2}-k_{3} e_{3} \\
& u_{4}(t)=\hat{d}\left(e_{1}+e_{2}\right)-k_{4} e_{4}
\end{aligned}
$$

where $\hat{a}, \hat{b}, \hat{c}$ and $\hat{d}$ are estimates of $a, b, c$ and $d$, respectively, and $k_{i},(i=1,2,3,4)$ are positive constants.

Substituting (7) into (6), the error dynamics simplifies to

$$
\begin{aligned}
& \dot{e}_{1}=(a-\hat{a})\left(e_{2}-e_{1}\right)-k_{1} e_{1} \\
& \dot{e}_{2}=(c-\hat{c}) e_{2}-k_{2} e_{2} \\
& \dot{e}_{3}=-(b-\hat{b}) e_{3}-k_{3} e_{3} \\
& \dot{e}_{4}=-(d-\hat{d})\left(e_{1}+e_{2}\right)-k_{4} e_{4}
\end{aligned}
$$

Let us now define the parameter estimation errors as 
Computer Science \& Engineering: An International Journal (CSEIJ), Vol.1, No.5, December 2011

$$
e_{a}=a-\hat{a}, e_{b}=b-\hat{b}, e_{c}=c-\hat{c} \text { and } e_{d}=d-\hat{d}
$$

Substituting (9) into (8), we obtain the error dynamics as

$$
\begin{aligned}
& \dot{e}_{1}=e_{a}\left(e_{2}-e_{1}\right)-k_{1} e_{1} \\
& \dot{e}_{2}=e_{c} e_{2}-k_{2} e_{2} \\
& \dot{e}_{3}=-e_{b} e_{3}-k_{3} e_{3} \\
& \dot{e}_{4}=-e_{d}\left(e_{1}+e_{2}\right)-k_{4} e_{4}
\end{aligned}
$$

For the derivation of the update law for adjusting the estimates of the parameters, the Lyapunov approach is used.

We consider the quadratic Lyapunov function defined by

$$
V\left(e_{1}, e_{2}, e_{3}, e_{4}, e_{a}, e_{b}, e_{c}, e_{d}\right)=\frac{1}{2}\left(e_{1}^{2}+e_{2}^{2}+e_{3}^{2}+e_{4}^{2}+e_{a}^{2}+e_{b}^{2}+e_{c}^{2}+e_{d}^{2}\right),
$$

which is a positive definite function on $R^{8}$.

We also note that

$$
\dot{e}_{a}=-\dot{\hat{a}}, \dot{e}_{b}=-\dot{\hat{b}}, \quad \dot{e}_{c}=-\dot{\hat{c}} \text { and } \quad \dot{e}_{d}=-\dot{\hat{d}}
$$

Differentiating (11) along the trajectories of (10) and using (12), we obtain

$$
\begin{aligned}
\dot{V}= & -k_{1} e_{1}^{2}-k_{2} e_{2}^{2}-k_{3} e_{3}^{2}-k_{4} e_{4}^{2}+e_{a}\left[e_{1}\left(e_{2}-e_{1}\right)-\dot{\hat{a}}\right]+e_{b}\left[-e_{3}^{2}-\dot{\hat{b}}\right] \\
& +e_{c}\left[e_{2}^{2}-\dot{\hat{c}}\right]+e_{d}\left[-e_{4}\left(e_{1}+e_{2}\right)-\dot{\hat{d}}\right]
\end{aligned}
$$

In view of Eq. (13), the estimated parameters are updated by the following law:

$$
\begin{aligned}
& \dot{\hat{a}}=e_{1}\left(e_{2}-e_{1}\right)+k_{5} e_{a} \\
& \dot{\hat{b}}=-e_{3}^{2}+k_{6} e_{b} \\
& \dot{\hat{c}}=e_{2}^{2}+k_{7} e_{c} \\
& \dot{\hat{d}}=-e_{4}\left(e_{1}+e_{2}\right)+k_{8} e_{d}
\end{aligned}
$$

where $k_{4}, k_{5}, k_{6}$ and $k_{7}$ are positive constants.

Substituting (14) into (13), we obtain

$$
\dot{V}=-k_{1} e_{1}^{2}-k_{2} e_{2}^{2}-k_{3} e_{3}^{2}-k_{4} e_{4}^{2}-k_{5} e_{a}^{2}-k_{6} e_{b}^{2}-k_{7} e_{c}^{2}-k_{8} e_{d}^{2}
$$

which is a negative definite function on $R^{8}$. 
Computer Science \& Engineering: An International Journal (CSEIJ), Vol.1, No.5, December 2011

Thus, by Lyapunov stability theory [35], it is immediate that the hybrid synchronization error $e_{i},(i=1,2,3,4)$ and the parameter estimation error $e_{a}, e_{b}, e_{c}, e_{d}$ decay to zero exponentially with time.

Hence, we have proved the following result.

Theorem 1. The identical hyperchaotic Pang systems (3) and (4) with unknown parameters are globally and exponentially synchronized via the adaptive control law (7), where the update law for the parameter estimates is given by $(14)$ and $k_{i},(i=1,2, \ldots, 8)$ are positive constants. Also, the parameter estimates $\hat{a}(t), \hat{b}(t), \hat{c}(t)$ and $\hat{d}(t)$ exponentially converge to the original values of the parameters $a, b, c$ and $d$, respectively, as $t \rightarrow \infty$.

\subsection{Numerical Results}

For the numerical simulations, the fourth-order Runge-Kutta method with time-step $h=10^{-6}$ is used to solve the hyperchaotic systems (3) and (4) with the adaptive control law (14) and the parameter update law (14) using MATLAB.

We take

$$
k_{i}=4 \text { for } i=1,2, \ldots, 8 \text {. }
$$

For the hyperchaotic Pang systems (3) and (4), the parameter values are taken as

$$
a=36, \quad b=3, \quad c=20, \quad d=2
$$

Suppose that the initial values of the parameter estimates are

$$
\hat{a}(0)=12, \hat{b}(0)=4, \hat{c}(0)=2, \hat{d}(0)=21
$$

The initial values of the master system (3) are taken as

$$
x_{1}(0)=12, \quad x_{2}(0)=18, \quad x_{3}(0)=35, \quad x_{4}(0)=6
$$

The initial values of the slave system (4) are taken as

$$
y_{1}(0)=20, y_{2}(0)=5, \quad y_{3}(0)=16, \quad y_{4}(0)=22
$$

Figure 3 depicts the global chaos synchronization of the identical hyperchaotic Pang systems (3) and (4).

Figure 4 shows that the estimated values of the parameters, viz. $\hat{a}(t), \hat{b}(t), \hat{c}(t)$ and $\hat{d}(t)$ converge exponentially to the system parameters

$$
a=36, b=3, c=20 \text { and } d=2
$$

as $t \rightarrow \infty$. 
Computer Science \& Engineering: An International Journal (CSEIJ), Vol.1, No.5, December 2011
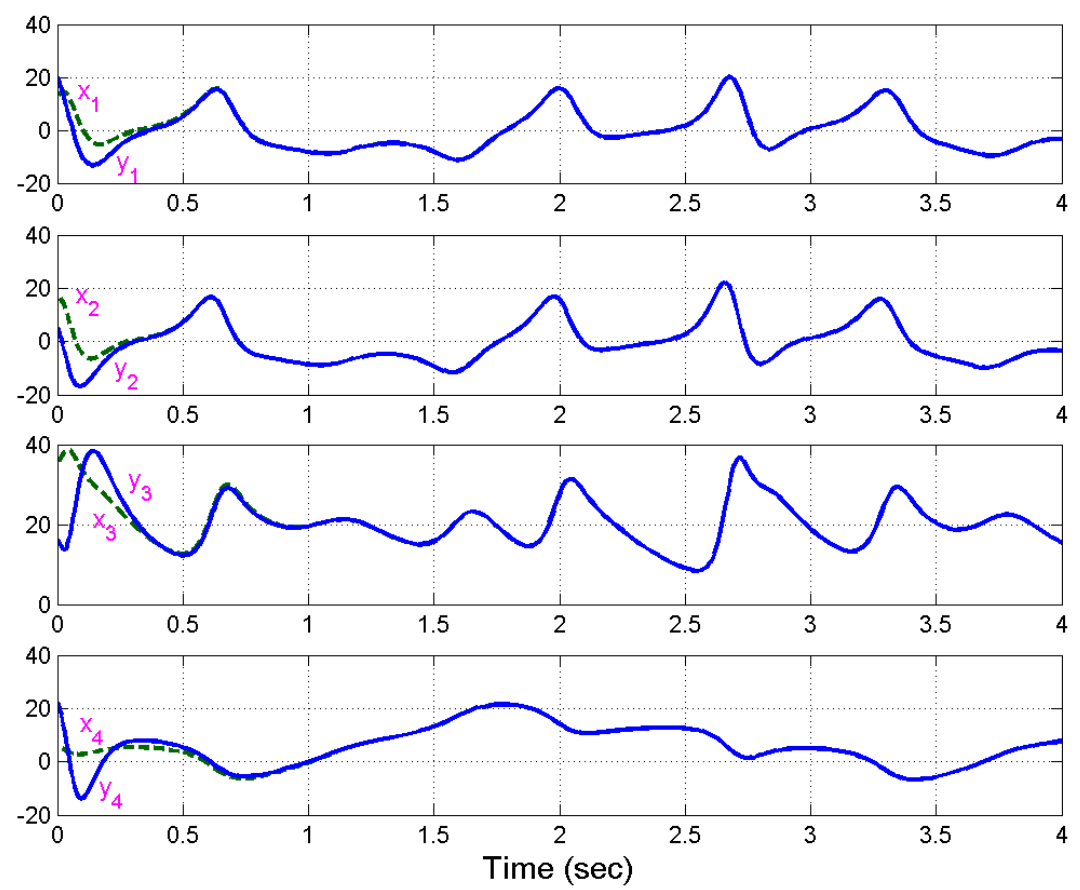

Figure 3. Complete Synchronization of Hyperchaotic Pang Systems

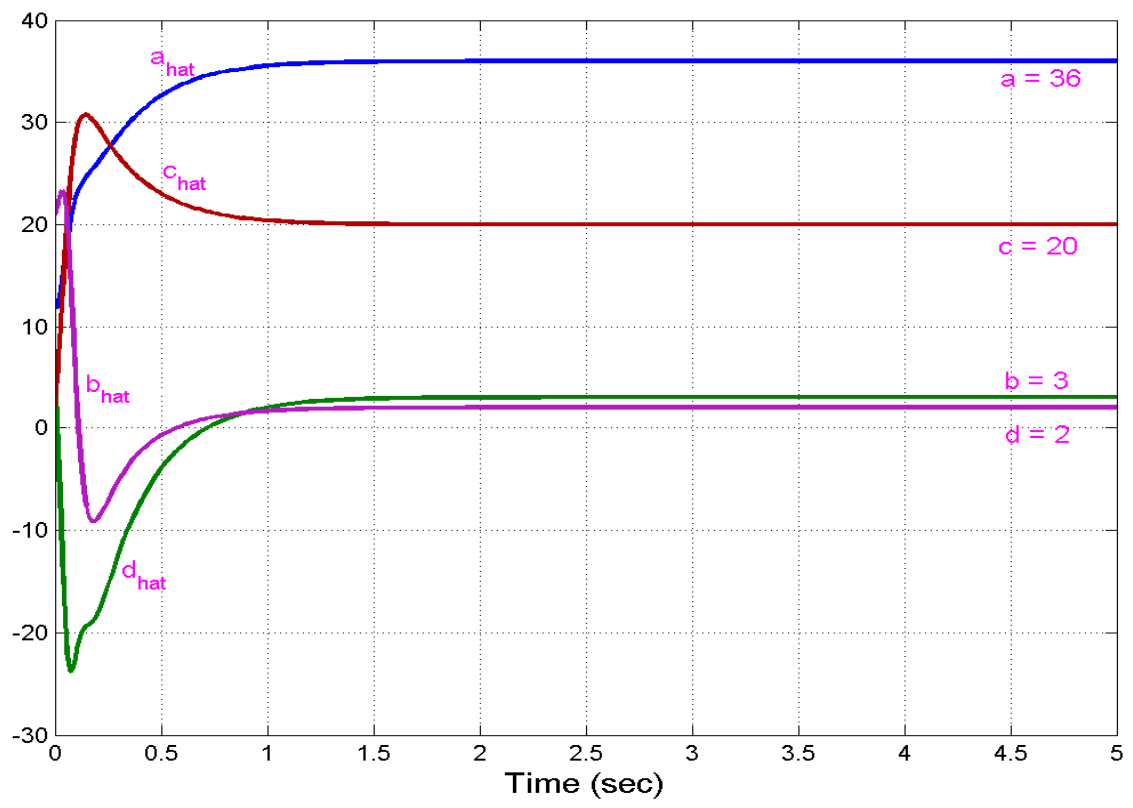

Figure 4. Parameter Estimates $\hat{a}(t), \hat{b}(t), \hat{c}(t), \hat{d}(t)$ 
Computer Science \& Engineering: An International Journal (CSEIJ), Vol.1, No.5, December 2011

\section{Adaptive Synchronization of Identical Hyperchaotic Wang SYSTEMS}

\subsection{Theoretical Results}

In this section, we deploy adaptive control to achieve new results for the global chaos synchronization of identical hyperchaotic Wang systems ([34], 2006), where the parameters of the master and slave systems are unknown.

As the master system, we consider the hyperchaotic Wang dynamics described by

$$
\begin{aligned}
& \dot{x}_{1}=\alpha\left(x_{2}-x_{1}\right) \\
& \dot{x}_{2}=\beta x_{1}-x_{1} x_{3}+x_{4} \\
& \dot{x}_{3}=-\gamma x_{3}+\varepsilon x_{1}^{2} \\
& \dot{x}_{4}=-\delta x_{1}
\end{aligned}
$$

where $x_{1}, x_{2}, x_{3}, x_{4}$ are the state variables and $\alpha, \beta, \gamma, \delta, \varepsilon$ are unknown, real ,constant parameters of the system.

As the slave system, we consider the controlled hyperchaotic Wang dynamics described by

$$
\begin{aligned}
& \dot{y}_{1}=\alpha\left(y_{2}-y_{1}\right)+u_{1} \\
& \dot{y}_{2}=\beta y_{1}-y_{1} y_{3}+y_{4}+u_{2} \\
& \dot{y}_{3}=-\gamma y_{3}+\varepsilon y_{1}^{2}+u_{3} \\
& \dot{y}_{4}=-\delta y_{1}+u_{4}
\end{aligned}
$$

where $y_{1}, y_{2}, y_{3}, y_{4}$ are the state variables and $u_{1}, u_{2}, u_{3}, u_{4}$ are the nonlinear controllers to be designed.

The chaos synchronization error is defined by

$$
\begin{aligned}
& e_{1}=y_{1}-x_{1} \\
& e_{2}=y_{2}-x_{2} \\
& e_{3}=y_{3}-x_{3} \\
& e_{4}=y_{4}-x_{4}
\end{aligned}
$$

The error dynamics is easily obtained as

$$
\begin{aligned}
& \dot{e}_{1}=\alpha\left(e_{2}-e_{1}\right)+u_{1} \\
& \dot{e}_{2}=\beta e_{1}+e_{4}-y_{1} y_{3}+x_{1} x_{3}+u_{2} \\
& \dot{e}_{3}=-\gamma e_{3}+\varepsilon\left(y_{1}^{2}-x_{1}^{2}\right)+u_{3} \\
& \dot{e}_{4}=-\delta e_{1}+u_{4}
\end{aligned}
$$

Let us now define the adaptive control functions 


$$
\begin{aligned}
& u_{1}(t)=-\hat{\alpha}\left(e_{2}-e_{1}\right)-k_{1} e_{1} \\
& u_{2}(t)=-\hat{\beta} e_{1}-e_{4}+y_{1} y_{3}-x_{1} x_{3}-k_{2} e_{2} \\
& u_{3}(t)=\hat{\gamma} e_{3}-\hat{\varepsilon}\left(y_{1}^{2}-x_{1}^{2}\right)-k_{3} e_{3} \\
& u_{4}(t)=\hat{\delta} e_{1}-k_{4} e_{4}
\end{aligned}
$$

where $\hat{\alpha}, \hat{\beta}, \hat{\gamma}, \hat{\delta}$ and $\hat{\varepsilon}$ are estimates of $\alpha, \beta, \gamma, \delta$ and $\varepsilon$, respectively, and $k_{i},(i=1,2,3,4)$ are positive constants.

Substituting (20) into (19), the error dynamics simplifies to

$$
\begin{aligned}
& \dot{e}_{1}=(\alpha-\hat{\alpha})\left(e_{2}-e_{1}\right)-k_{1} e_{1} \\
& \dot{e}_{2}=(\beta-\hat{\beta}) e_{1}-k_{2} e_{2} \\
& \dot{e}_{3}=-(\gamma-\hat{\gamma}) e_{3}+(\varepsilon-\hat{\varepsilon})\left(y_{1}^{2}-x_{1}^{2}\right)-k_{3} e_{3} \\
& \dot{e}_{4}=-(\delta-\hat{\delta}) e_{1}-k_{4} e_{4}
\end{aligned}
$$

Let us now define the parameter estimation errors as

$$
e_{\alpha}=\alpha-\hat{\alpha}, e_{\beta}=\beta-\hat{\beta}, e_{\gamma}=\gamma-\hat{\gamma}, e_{\delta}=\delta-\hat{\delta} \text { and } e_{\varepsilon}=\varepsilon-\hat{\varepsilon}
$$

Substituting (22) into (21), we obtain the error dynamics as

$$
\begin{aligned}
& \dot{e}_{1}=e_{\alpha}\left(e_{2}-e_{1}\right)-k_{1} e_{1} \\
& \dot{e}_{2}=e_{\beta} e_{1}-k_{2} e_{2} \\
& \dot{e}_{3}=-e_{\gamma} e_{3}+e_{\varepsilon}\left(y_{1}^{2}-x_{1}^{2}\right)-k_{3} e_{3} \\
& \dot{e}_{4}=-e_{\delta} e_{1}-k_{4} e_{4}
\end{aligned}
$$

For the derivation of the update law for adjusting the estimates of the parameters, the Lyapunov approach is used.

We consider the quadratic Lyapunov function defined by

$$
V\left(e_{1}, e_{2}, e_{3}, e_{4}, e_{\alpha}, e_{\beta}, e_{\gamma}, e_{\delta}, e_{\varepsilon}\right)=\frac{1}{2}\left(e_{1}^{2}+e_{2}^{2}+e_{3}^{2}+e_{4}^{2}+e_{\alpha}^{2}+e_{\beta}^{2}+e_{\gamma}^{2}+e_{\delta}^{2}+e_{\varepsilon}^{2}\right),
$$

which is a positive definite function on $R^{9}$.

We also note that

$$
\dot{e}_{\alpha}=-\dot{\hat{\alpha}}, \dot{e}_{\beta}=-\dot{\hat{\beta}}, \dot{e}_{\gamma}=-\dot{\hat{\gamma}}, \dot{e}_{\delta}=-\dot{\hat{\delta}} \quad \text { and } \quad \dot{e}_{\varepsilon}=-\dot{\hat{\varepsilon}}
$$

Differentiating (24) along the trajectories of (23) and using (25), we obtain 
Computer Science \& Engineering: An International Journal (CSEIJ), Vol.1, No.5, December 2011

$$
\begin{aligned}
\dot{V}= & -k_{1} e_{1}^{2}-k_{2} e_{2}^{2}-k_{3} e_{3}^{2}-k_{4} e_{4}^{2}+e_{\alpha}\left[e_{1}\left(e_{2}-e_{1}\right)-\dot{\hat{\alpha}}\right]+e_{\beta}\left[e_{1} e_{2}-\dot{\hat{\beta}}\right] \\
& +e_{\gamma}\left[-e_{3}^{2}-\dot{\hat{\gamma}}\right]+e_{\delta}\left[-e_{1} e_{4}-\dot{\hat{\delta}}\right]+e_{\varepsilon}\left[e_{3}\left(y_{1}^{2}-x_{1}^{2}\right)-\dot{\hat{\varepsilon}}\right]
\end{aligned}
$$

In view of Eq. (26), the estimated parameters are updated by the following law:

$$
\begin{aligned}
& \dot{\hat{\alpha}}=e_{1}\left(e_{2}-e_{1}\right)+k_{5} e_{\alpha} \\
& \dot{\hat{\beta}}=e_{1} e_{2}+k_{6} e_{\beta} \\
& \dot{\hat{\gamma}}=-e_{3}^{2}+k_{7} e_{\gamma} \\
& \dot{\hat{\delta}}=-e_{1} e_{4}+k_{8} e_{\delta} \\
& \dot{\hat{\varepsilon}}=e_{3}\left(y_{1}^{2}-x_{1}^{2}\right)+k_{9} e_{\varepsilon}
\end{aligned}
$$

where $k_{i},(i=5, \ldots, 9)$ are positive constants.

Substituting (27) into (26), we obtain

$$
\dot{V}=-k_{1} e_{1}^{2}-k_{2} e_{2}^{2}-k_{3} e_{3}^{2}-k_{4} e_{4}^{2}-k_{5} e_{\alpha}^{2}-k_{6} e_{\beta}^{2}-k_{7} e_{\gamma}^{2}-k_{8} e_{\delta}^{2}-k_{9} e_{\varepsilon}^{2}
$$

which is a negative definite function on $R^{9}$.

Thus, by Lyapunov stability theory [35], it is immediate that the hybrid synchronization error $e_{i},(i=1,2,3,4)$ and the parameter estimation error $e_{\alpha}, e_{\beta}, e_{\gamma}, e_{\delta}, e_{\varepsilon}$ decay to zero exponentially with time.

Hence, we have proved the following result.

Theorem 2. The identical hyperchaotic Wang systems (16) and (17) with unknown parameters are globally and exponentially synchronized via the adaptive control law (20), where the update law for the parameter estimates is given by (27) and $k_{i},(i=1,2, \ldots, 9)$ are positive constants.

Also, the parameter estimates $\hat{\alpha}(t), \hat{\beta}(t), \hat{\gamma}(t), \hat{\delta}(t)$ and $\hat{\mathcal{E}}(t)$ exponentially converge to the original values of the parameters $\alpha, \beta, \gamma, \delta$ and $\varepsilon$, respectively, as $t \rightarrow \infty$.

\subsection{Numerical Results}

For the numerical simulations, the fourth-order Runge-Kutta method with time-step $h=10^{-6}$ is used to solve the hyperchaotic systems (16) and (17) with the adaptive control law (20) and the parameter update law (27) using MATLAB.

We take

$$
k_{i}=4 \text { for } i=1,2, \ldots, 9
$$

For the hyperchaotic Wang systems (16) and (17), the parameter values are taken as 
Computer Science \& Engineering: An International Journal (CSEIJ), Vol.1, No.5, December 2011

$$
\alpha=10, \quad \beta=40, \quad \gamma=2.5, \quad \delta=10.6, \quad \varepsilon=4
$$

Suppose that the initial values of the parameter estimates are

$$
\hat{\alpha}(0)=5, \quad \hat{\beta}(0)=10, \quad \hat{\gamma}(0)=7, \quad \hat{\delta}(0)=14, \quad \hat{\varepsilon}(0)=9
$$

The initial values of the master system (16) are taken as

$$
x_{1}(0)=21, \quad x_{2}(0)=7, x_{3}(0)=16, x_{4}(0)=18
$$

The initial values of the slave system (17) are taken as

$$
y_{1}(0)=4, y_{2}(0)=25, y_{3}(0)=30, y_{4}(0)=11
$$

Figure 5 depicts the global chaos synchronization of the identical hyperchaotic Wang systems (16) and (17).
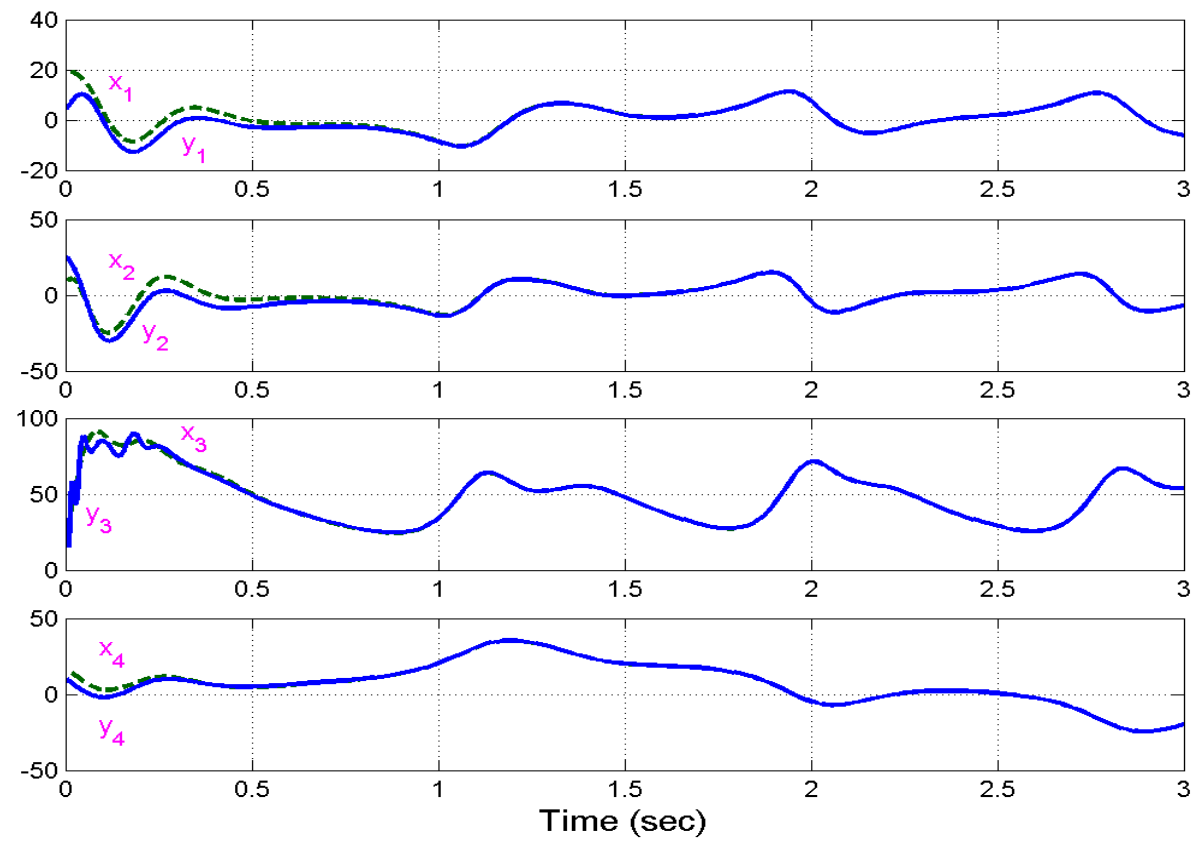

Figure 5. Complete Synchronization of Hyperchaotic Wang Systems

Figure 6 shows that the estimated values of the parameters, viz. $\hat{\alpha}(t), \hat{\beta}(t), \hat{\gamma}(t), \hat{\delta}(t)$ and $\hat{\varepsilon}(t)$ converge exponentially to the system parameters $\alpha=10, \beta=40, \gamma=2.5, \delta=10.6$ and $\varepsilon=4$ as $t \rightarrow \infty$. 
Computer Science \& Engineering: An International Journal (CSEIJ), Vol.1, No.5, December 2011

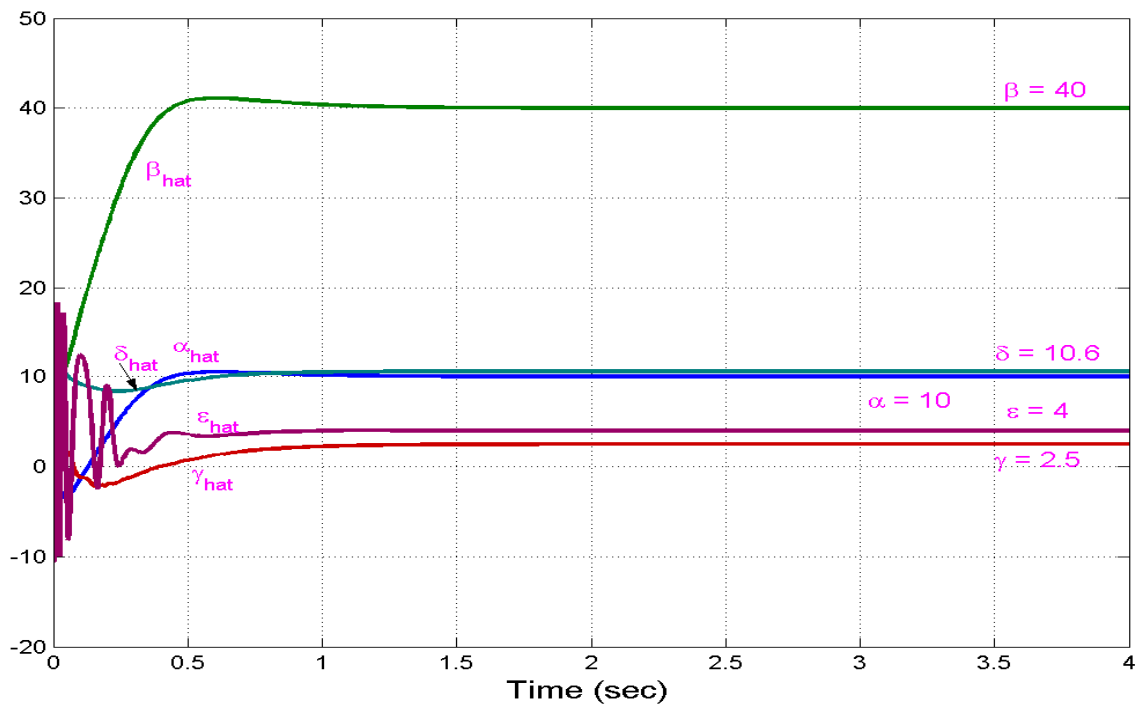

Figure 6. Parameter Estimates $\hat{\alpha}(t), \hat{\beta}(t), \hat{\gamma}(t), \hat{\delta}(t), \hat{\varepsilon}(t)$

\section{Adaptive Synchronization of Hyperchaotic Pang and HYPERCHAOTIC WANG SYSTEMS}

\subsection{Theoretical Results}

In this section, we discuss the global chaos synchronization of non-identical hyperchaotic Pang system ([33], 2011) and hyperchaotic Wang system ([34], 2006), where the parameters of the master and slave systems are unknown.

As the master system, we consider the hyperchaotic Pang system described by

$$
\begin{aligned}
& \dot{x}_{1}=a\left(x_{2}-x_{1}\right) \\
& \dot{x}_{2}=c x_{2}-x_{1} x_{3}+x_{4} \\
& \dot{x}_{3}=-b x_{3}+x_{1} x_{2} \\
& \dot{x}_{4}=-d\left(x_{1}+x_{2}\right)
\end{aligned}
$$

where $x_{1}, x_{2}, x_{3}, x_{4}$ are the state variables and $a, b, c, d$ are unknown, real ,constant parameters of the system.

As the slave system, we consider the controlled hyperchaotic Wang dynamics described by

$$
\begin{aligned}
& \dot{y}_{1}=\alpha\left(y_{2}-y_{1}\right)+u_{1} \\
& \dot{y}_{2}=\beta y_{1}-y_{1} y_{3}+y_{4}+u_{2} \\
& \dot{y}_{3}=-\gamma y_{3}+\varepsilon y_{1}^{2}+u_{3} \\
& \dot{y}_{4}=-\delta y_{1}+u_{4}
\end{aligned}
$$


Computer Science \& Engineering: An International Journal (CSEIJ), Vol.1, No.5, December 2011 where $y_{1}, y_{2}, y_{3}, y_{4}$ are the state variables $, \alpha, \beta, \gamma, \delta, \varepsilon$ are unknown, real, constant parameters of the system and $u_{1}, u_{2}, u_{3}, u_{4}$ are the nonlinear controllers to be designed.

The synchronization error is defined by

$$
\begin{aligned}
& e_{1}=y_{1}-x_{1} \\
& e_{2}=y_{2}-x_{2} \\
& e_{3}=y_{3}-x_{3} \\
& e_{4}=y_{4}-x_{4}
\end{aligned}
$$

The error dynamics is easily obtained as

$$
\begin{aligned}
& \dot{e}_{1}=\alpha\left(y_{2}-y_{1}\right)-a\left(x_{2}-x_{1}\right)+u_{1} \\
& \dot{e}_{2}=\beta y_{1}-c x_{2}+e_{4}-y_{1} y_{3}+x_{1} x_{3}+u_{2} \\
& \dot{e}_{3}=-\gamma y_{3}+b x_{3}+\varepsilon y_{1}^{2}-x_{1} x_{2}+u_{3} \\
& \dot{e}_{4}=-\delta y_{1}+d\left(x_{1}+x_{2}\right)+u_{4}
\end{aligned}
$$

Let us now define the adaptive control functions

$$
\begin{aligned}
& u_{1}(t)=-\hat{\alpha}\left(y_{2}-y_{1}\right)+\hat{a}\left(x_{2}-x_{1}\right)-k_{1} e_{1} \\
& u_{2}(t)=-\hat{\beta} y_{1}+\hat{c} x_{2}-e_{4}+y_{1} y_{3}-x_{1} x_{3}-k_{2} e_{2} \\
& u_{3}(t)=\hat{\gamma} y_{3}-\hat{b} x_{3}-\hat{\varepsilon} y_{1}^{2}+x_{1} x_{2}-k_{3} e_{3} \\
& u_{4}(t)=\hat{\delta} y_{1}-\hat{d}\left(x_{1}+x_{2}\right)-k_{4} e_{4}
\end{aligned}
$$

where $\hat{a}, \hat{b}, \hat{c}, \hat{d}, \hat{\alpha}, \hat{\beta}, \hat{\gamma}, \hat{\delta}$ and $\hat{\varepsilon}$ are estimates of $a, b, c, d, \alpha, \beta, \gamma, \delta$ and $\varepsilon$, respectively, and $k_{i},(i=1,2,3,4)$ are positive constants.

Substituting (33) into (32), the error dynamics simplifies to

$$
\begin{aligned}
& \dot{e}_{1}=(\alpha-\hat{\alpha})\left(y_{2}-y_{1}\right)-(a-\hat{a})\left(x_{2}-x_{1}\right)-k_{1} e_{1} \\
& \dot{e}_{2}=(\beta-\hat{\beta}) y_{1}-(c-\hat{c}) x_{2}-k_{2} e_{2} \\
& \dot{e}_{3}=-(\gamma-\hat{\gamma}) y_{3}+(b-\hat{b}) x_{3}+(\varepsilon-\hat{\varepsilon}) y_{1}^{2}-k_{3} e_{3} \\
& \dot{e}_{4}=-(\delta-\hat{\delta}) y_{1}+(d-\hat{d})\left(x_{1}+x_{2}\right)-k_{4} e_{4}
\end{aligned}
$$

Let us now define the parameter estimation errors as

$$
\begin{aligned}
& e_{a}=a-\hat{a}, \quad e_{b}=b-\hat{b}, \quad e_{c}=c-\hat{c}, \quad e_{d}=d-\hat{d} \\
& e_{\alpha}=\alpha-\hat{\alpha}, \quad e_{\beta}=\beta-\hat{\beta}, \quad e_{\gamma}=\gamma-\hat{\gamma}, e_{\delta}=\delta-\hat{\delta}, e_{\varepsilon}=\varepsilon-\hat{\varepsilon}
\end{aligned}
$$

Substituting (35) into (34), we obtain the error dynamics as 
Computer Science \& Engineering: An International Journal (CSEIJ), Vol.1, No.5, December 2011

$$
\begin{aligned}
& \dot{e}_{1}=e_{\alpha}\left(y_{2}-y_{1}\right)-e_{a}\left(x_{2}-x_{1}\right)-k_{1} e_{1} \\
& \dot{e}_{2}=e_{\beta} y_{1}-e_{c} x_{2}-k_{2} e_{2} \\
& \dot{e}_{3}=-e_{\gamma} y_{3}+e_{b} x_{3}+e_{\varepsilon} y_{1}^{2}-k_{3} e_{3} \\
& \dot{e}_{4}=-e_{\delta} y_{1}+e_{d}\left(x_{1}+x_{2}\right)-k_{4} e_{4}
\end{aligned}
$$

We consider the quadratic Lyapunov function defined by

$$
V=\frac{1}{2}\left(e_{1}^{2}+e_{2}^{2}+e_{3}^{2}+e_{4}^{2}+e_{a}^{2}+e_{b}^{2}+e_{c}^{2}+e_{d}^{2}+e_{\alpha}^{2}+e_{\beta}^{2}+e_{\gamma}^{2}+e_{\delta}^{2}+e_{\varepsilon}^{2}\right),
$$

which is a positive definite function on $R^{13}$.

We also note that

$$
\begin{aligned}
& \dot{e}_{a}=-\dot{\hat{a}}, \quad \dot{e}_{b}=-\dot{\hat{b}}, \quad \dot{e}_{c}=-\dot{\hat{\hat{c}}}, \dot{e}_{d}=-\dot{\hat{\hat{d}}} \\
& \dot{e}_{\alpha}=-\dot{\hat{\alpha}}, \dot{e}_{\beta}=-\dot{\hat{\beta}}, \dot{e}_{\gamma}=-\dot{\hat{\gamma}}, \dot{e}_{\delta}=-\dot{\hat{\delta}}, \dot{e}_{\varepsilon}=-\dot{\hat{\varepsilon}}
\end{aligned}
$$

Differentiating (37) along the trajectories of (36) and using (38), we obtain

$$
\begin{aligned}
\dot{V}= & -k_{1} e_{1}^{2}-k_{2} e_{2}^{2}-k_{3} e_{3}^{2}-k_{4} e_{4}^{2}+e_{a}\left[-e_{1}\left(x_{2}-x_{1}\right)-\dot{\hat{a}}\right]+e_{b}\left[e_{3} x_{3}-\dot{\hat{b}}\right]+e_{c}\left[-e_{2} x_{2}-\dot{\hat{c}}\right] \\
& +e_{d}\left[e_{4}\left(x_{1}+x_{2}\right)-\dot{\hat{d}}\right]+e_{\alpha}\left[e_{1}\left(y_{2}-y_{1}\right)-\dot{\hat{\alpha}}\right]+e_{\beta}\left[e_{2} y_{1}-\dot{\hat{\beta}}\right]+e_{\gamma}\left[-e_{3} y_{3}-\dot{\hat{\gamma}}\right] \\
& +e_{\delta}\left[-e_{4} y_{1}-\dot{\hat{\delta}}\right]+e_{\varepsilon}\left[e_{3} y_{1}^{2}-\dot{\hat{\varepsilon}}\right]
\end{aligned}
$$

In view of Eq. (39), the estimated parameters are updated by the following law:

$$
\begin{array}{ll}
\dot{\hat{a}}=-e_{1}\left(x_{2}-x_{1}\right)+k_{5} e_{a}, & \dot{\hat{\alpha}}=e_{1}\left(y_{2}-y_{1}\right)+k_{9} e_{\alpha} \\
\dot{\hat{b}}=e_{3} x_{3}+k_{6} e_{b}, & \dot{\hat{\beta}}=e_{2} y_{1}+k_{10} e_{\beta} \\
\dot{\hat{c}}=-e_{2} x_{2}+k_{7} e_{c}, & \dot{\hat{\gamma}}=-e_{3} y_{3}+k_{11} e_{\gamma} \\
\dot{\hat{d}}=e_{4}\left(x_{1}+x_{2}\right)+k_{8} e_{d}, & \dot{\hat{\delta}}=-e_{4} y_{1}+k_{12} e_{\delta} \\
& \dot{\hat{\varepsilon}}=e_{3} y_{1}^{2}+k_{13} e_{\varepsilon}
\end{array}
$$

where $k_{i},(i=5, \ldots, 13)$ are positive constants.

Substituting (40) into (39), we obtain

$$
\begin{aligned}
\dot{V}= & -k_{1} e_{1}^{2}-k_{2} e_{2}^{2}-k_{3} e_{3}^{2}-k_{4} e_{4}^{2}-k_{5} e_{a}^{2}-k_{6} e_{b}^{2}-k_{7} e_{c}^{2}-k_{8} e_{d}^{2}-k_{9} e_{\alpha}^{2}-k_{10} e_{\beta}^{2} \\
& -k_{11} e_{\gamma}^{2}-k_{12} e_{\delta}^{2}-k_{13} e_{\varepsilon}^{2}
\end{aligned}
$$

which is a negative definite function on $R^{13}$. 
Computer Science \& Engineering: An International Journal (CSEIJ), Vol.1, No.5, December 2011

Thus, by Lyapunov stability theory [35], it is immediate that the hybrid synchronization error $e_{i},(i=1,2,3,4)$ and all the parameter estimation errors decay to zero exponentially with time.

Hence, we have proved the following result.

Theorem 3. The non-identical hyperchaotic Pang system (29) and hyperchaotic Wang system (30) with unknown parameters are globally and exponentially synchronized via the adaptive control law (33), where the update law for the parameter estimates is given by (40) and $k_{i},(i=1,2, \ldots, 13)$ are positive constants. Also, the parameter estimates $\hat{a}(t), \hat{b}(t), \hat{c}(t), \hat{d}(t), \hat{\alpha}(t), \hat{\beta}(t), \hat{\gamma}(t), \hat{\delta}(t)$ and $\hat{\varepsilon}(t)$ exponentially converge to the original values of the parameters $a, b, c, d, \alpha, \beta, \gamma, \delta$ and $\varepsilon$, respectively, as $t \rightarrow \infty$.

\subsection{Numerical Results}

For the numerical simulations, the fourth-order Runge-Kutta method with time-step $h=10^{-6}$ is used to solve the hyperchaotic systems (29) and (30) with the adaptive control law (33) and the parameter update law (40) using MATLAB. We take $k_{i}=4$ for $i=1,2, \ldots, 13$. For the hyperchaotic Pang and hyperchaotic Wang systems, the parameters of the systems are chosen so that the systems are hyperchaotic (see Section 2).

Suppose that the initial values of the parameter estimates are

$$
\begin{aligned}
& \hat{a}(0)=2, \hat{b}(0)=5, \hat{c}(0)=10, \hat{d}(0)=12 \\
& \hat{\alpha}(0)=7, \hat{\beta}(0)=9, \hat{\gamma}(0)=15, \hat{\delta}(0)=22, \hat{\varepsilon}(0)=25
\end{aligned}
$$

The initial values of the master system (29) are taken as

$$
x_{1}(0)=27, x_{2}(0)=11, x_{3}(0)=28, x_{4}(0)=6
$$

The initial values of the slave system (30) are taken as

$$
y_{1}(0)=10, \quad y_{2}(0)=26, \quad y_{3}(0)=9, y_{4}(0)=30
$$

Figure 7 depicts the global chaos synchronization of hyperchaotic Pang and hyperchaotic Wang systems. Figure 8 shows that the estimated values of the parameters, viz. $\hat{a}(t), \hat{b}(t), \hat{c}(t), \hat{d}(t)$, $\hat{\alpha}(t), \quad \hat{\beta}(t), \quad \hat{\gamma}(t), \quad \hat{\delta}(t)$ and $\hat{\varepsilon}(t)$ converge exponentially to the system parameters $a=36, b=3, c=20, d=2, \alpha=10, \beta=40, \gamma=2.5, \delta=10.6$ and $\varepsilon=4$, respectively, as $t \rightarrow \infty$. 
Computer Science \& Engineering: An International Journal (CSEIJ), Vol.1, No.5, December 2011
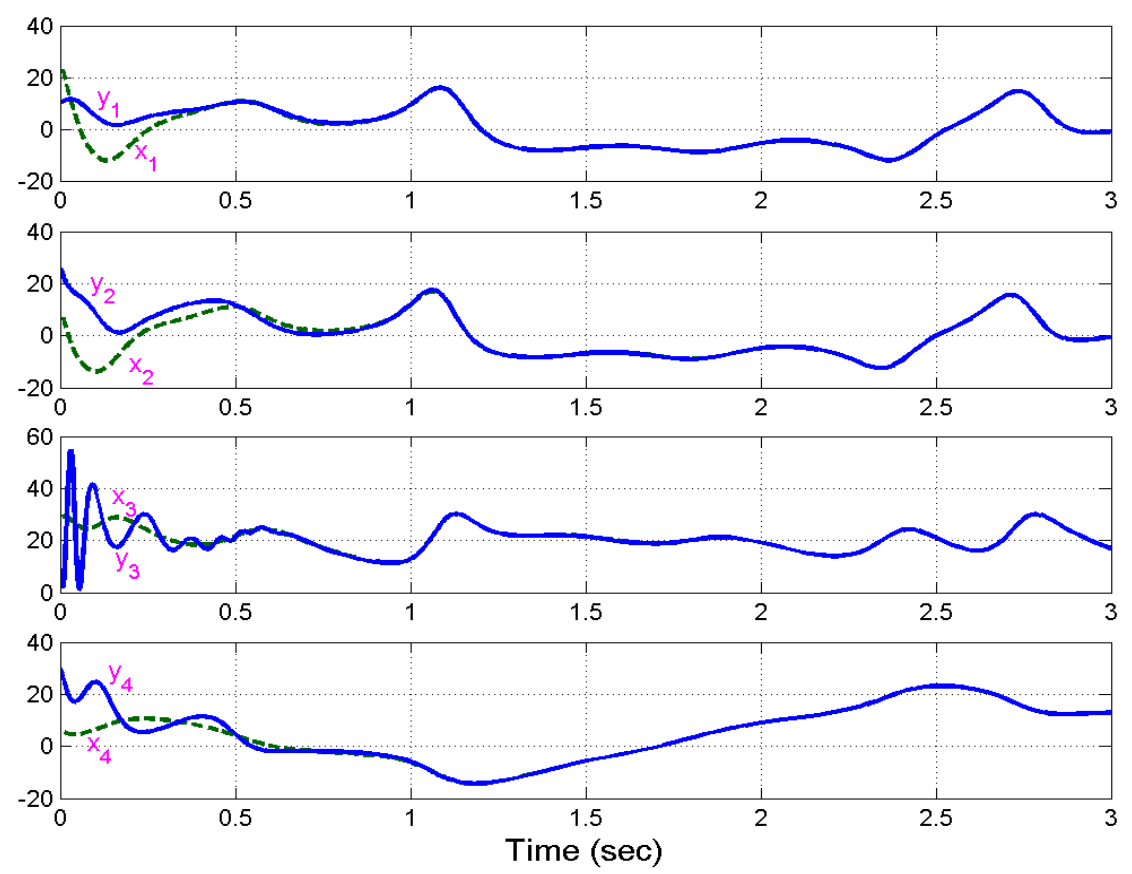

Figure 7. Complete Synchronization of Hyperchaotic Pang and Wang Systems

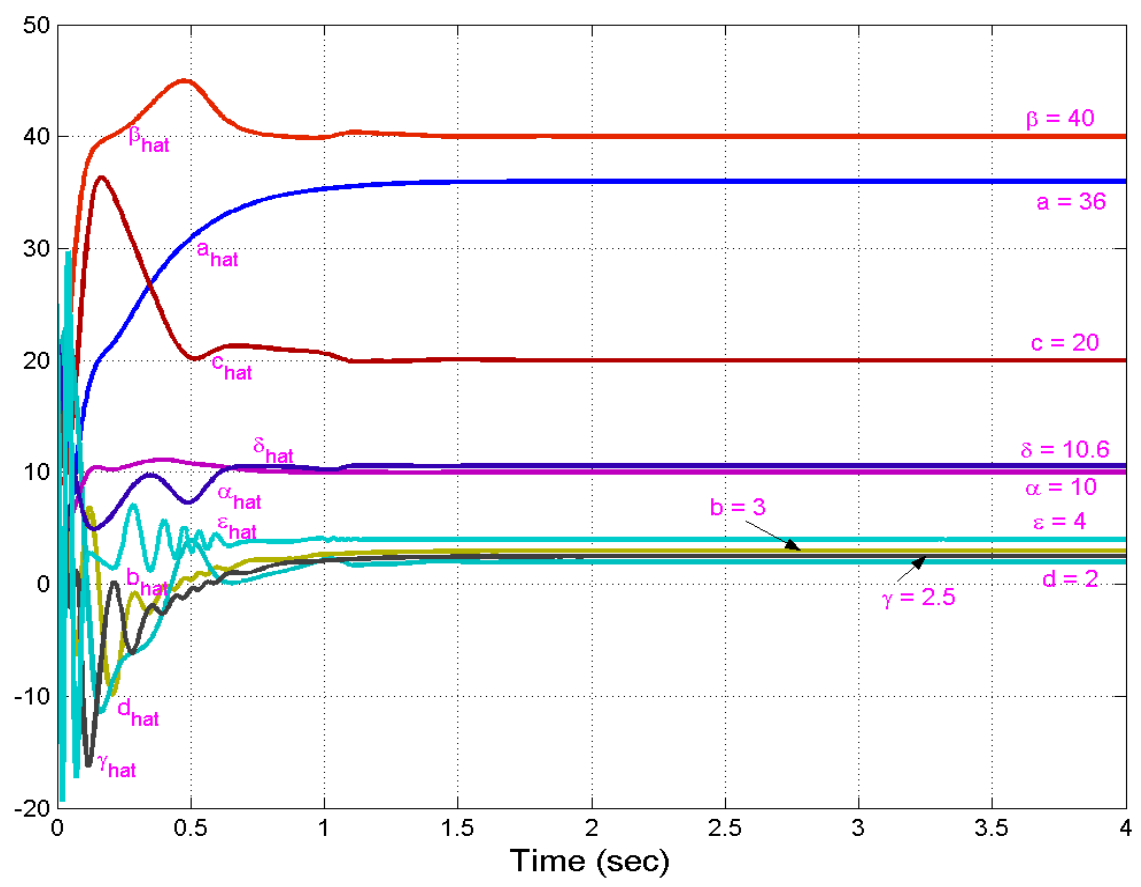

Figure 8. Parameter Estimates $\hat{a}(t), \hat{b}(t), \hat{c}(t), \hat{d}(t), \hat{\alpha}(t), \hat{\beta}(t), \hat{\gamma}(t)$ 
Computer Science \& Engineering: An International Journal (CSEIJ), Vol.1, No.5, December 2011

\section{CONCLUSIONS}

In this paper, we have derived new results for the adaptive synchronization of identical hyperchaotic Pang systems (2011), identical hyperchaotic Wang systems (2006) and non-identical hyperchaotic Pang and hyperchaotic Wang systems with unknown parameters. The adaptive synchronization results derived in this paper are established using Lyapunov stability theory. Since the Lyapunov exponents are not required for these calculations, the adaptive control method is a very effective and convenient for achieving global chaos synchronization for the uncertain hyperchaotic systems discussed in this paper. Numerical simulations are given to illustrate the effectiveness of the adaptive synchronization schemes derived in this paper for the global chaos synchronization of identical and non-identical uncertain hyperchaotic Pang and hyperchaotic Wang systems.

\section{REFERENCES}

[1] Alligood, K.T., Sauer, T. \& Yorke, J.A. (1997) Chaos: An Introduction to Dynamical Systems, Springer, New York.

[2] Lorenz, E.N. (1963) "Deterministic nonperiodic flow," Journal of the Atmospheric Sciences, Vol. 20, pp 130-141.

[3] Rössler, O.E. (1979) “An equation for hyperchaos,” Physics Letters A, Vol. 71, pp 151-157.

[4] Pecora, L.M. \& Carroll, T.L. (1990) "Synchronization in chaotic systems," Physical Review Letters, Vol. 64, pp 821-824.

[5] Fabiny, L. \& Wiesenfield, K. (1991) "Clustering behaviour of oscillator arrays," Physical Review A, Vol. 43, pp 2640-2648.

[6] Niu, H., Zhang, Q. \& Zhang, Y. (2002) "The chaos synchronization of a singular chemical model and a Williamowski-Rossler model," International Journal of Information and Systems Sciences, Vol. 6, No. 4, pp 355-364.

[7] Blasius, B., Huppert, A. \& Stone, L. (1999) "Complex dynamics and phase synchronization in spatially extended ecological system”, Nature, Vol. 399, pp 354-359.

[8] Kocarev, L. \& Parlitz, U. (1995) "General approach for chaotic synchronization with applications to communication," Physical Review Letters, Vol. 74, pp 5028-5030.

[9] Boccaletti, S., Farini, A. \& Arecchi, F.T. (1997) "Adaptive synchronization of chaos for secure communication," Physical Review E, Vol. 55, No. 5,

[10] Tao, Y. (1999) "Chaotic secure communication systems - history and new results," Telecommun. Review, Vol. 9, pp 597-634.

[11] Ott, E., Grebogi, C. \& Yorke, J.A. (1990) “Controlling chaos”, Physical Review Letters, Vol. 64, pp 1196-1199.

[12] Ho, M.C. \& Hung, Y.C. (2002) "Synchronization of two different chaotic systems using generalized active network," Physics Letters A, Vol. 301, pp 424-428.

[13] Huang, L., Feng, R. \& Wang, M. (2005) "Synchronization of chaotic systems via nonlinear control," Physical Letters A, Vol. 320, pp 271-275.

[14] Chen, H.K. (2005) "Global chaos synchronization of new chaotic systems via nonlinear control," Chaos, Solitons \& Fractals, Vol. 23, pp 1245-1251.

[15] Sundarapandian, V. \& Suresh, R. (2011) "Global chaos synchronization of hyperchaotic Qi and Jia systems by nonlinear control," International Journal of Distributed and Parallel Systems, Vol. 2, No. 2, pp 83-94.

[16] Sundarapandian, V. \& Karthikeyan, R. (2011) "Active controller design for global chaos antisynchronization of Li and Tigan chaotic systems," International Journal of Information Technology and Computer Science, vol. 3, no. 4, pp 255-268.

[17] Lu, J., Wu, X., Han, X. \& Lü, J. (2004) “Adaptive feedback synchronization of a unified chaotic system," Physics Letters A, Vol. 329, pp 327-333.

[18] Sundarapandian, V. (2011) "Adaptive control and synchronization of hyperchaotic Liu system," International Journal of Computer Science, Engineering and Information Technology, Vol. 1, No. 2, pp 29-40. 
Computer Science \& Engineering: An International Journal (CSEIJ), Vol.1, No.5, December 2011

[19] Sundarapandian, V. (2011) "Adaptive control and synchronization of hyperchaotic Newton-Leipnik system," International Journal of Advanced Information Technology, Vol. 1, No. 3, pp 22-33.

[20] Sundarapandian, V. (2011) "Adaptive synchronization of hyperchaotic Lorenz and hyperchaotic Lü systems," International Journal of Instrumentation and Control Systems, Vol. 1, No. 1, pp 1-18.

[21] Sundarapandian, V. (2011) "Adaptive control and synchronization of hyperchaotic Cai system," International Journal of Control Theory and Computer Modeling, Vol. 1, No. 1, pp 1-13.

[22] Sundarapandian, V. (2011) "Adaptive control and synchronization of Liu's four-wing chaotic system with cubic nonlinearity," International Journal of Computer Science, Engineering and Applications, Vol. 1, No. 4, pp 127-138.

[23] Zhao, J. \& Lu, J. (2006) "Using sampled-data feedback control and linear feedback synchronization in a new hyperchaotic system," Chaos, Solitons and Fractals, Vol. 35, pp 376-382.

[24] Park, J.H. \& Kwon, O.M. (2003) "A novel criterion for delayed feedback control of time-delay chaotic systems," Chaos, Solitons \& Fractals, Vol. 17, pp 709-716.

[25] Tan, X., Zhang, J. \& Yang, Y. (2003) "Synchronizing chaotic systems using backstepping design," Chaos, Solitons \& Fractals, Vol. 16, pp. 37-45.

[26] Vincent, U.E. (2008) "Chaotic synchronization using active control and backstepping control: A comparative analysis," Nonlinear Analysis: Modelling and Control, Vol. 13, No. 2, pp 253-261.

[27] Slotine, J.E. \& Sastry, S.S. (1983) "Tracking control of nonlinear systems using sliding surface with application to robotic manipulators," Internat. J. Control, Vol. 38, pp 465-492.

[28] Utkin, V.I. (1993) "Sliding mode control design principles and applications to electric drives," IEEE Trans. Industrial Electronics, Vol. 40, pp 23-36, 1993.

[29] Sundarapandian, V. \& S. Sivaperumal (2011) "Anti-synchronization of hyperchaotic Lorenz systems by sliding mode control," International Journal on Computer Science and Engineering, Vol. 3, No. 6, pp 2438-2449.

[30] Sundarapandian, V. (2011) "Sliding mode controller design for synchronization of Shimizu-Morioka chaotic systems," International Journal of Information Sciences and Techniques, Vol. 1, No. 1, pp 20-29.

[31] Sundarapandian, V. (2011) "Global chaos synchronization of four-wing chaotic systems by sliding mode control," International Journal of Control Theory and Computer Modeling, Vol. 1, No. 1, pp 15-31.

[32] Sundarapandian, V. (2011) "Global chaos synchronization of hyperchaotic Newton-Leipnik systems by sliding mode control," International Journal of Information Technology, Convergence and Services, Vol. 1, No. 4, pp 34-43.

[33] Pang, S. \& Liu, Y. (2011) "A new hyperchaotic system from the Lü system and its control," Journal of Computational and Applied Mathematics, Vol. 235, pp 2775-2789.

[34] Wang, F.Q. \& Liu, C.X. (2006) "Hyperchaos evolved from the Liu chaotic system," Chinese Physics, Vol. 15, pp 963-968.

[35] Hahn, W. (1967) The Stability of Motion, Springer, New York. 
Computer Science \& Engineering: An International Journal (CSEIJ), Vol.1, No.5, December 2011

\section{Authors}

Dr. V. Sundarapandian obtained his Doctor of Science degree in Electrical and Systems Engineering from Washington University, Saint Louis, USA under the guidance of Late Dr. Christopher I. Byrnes (Dean, School of Engineering and Applied Science) in 1996. He is currently Professor in the Research and Development Centre at Vel Tech Dr. RR \& Dr. SR Technical University, Chennai, Tamil Nadu, India. He has published over 190 refereed international publications. He has published over 100 papers in National Conferences and over 50 papers in International Conferences. He is the Editor-in-Chief of International Journal of Mathematics and Scientific Computing, International Journal of

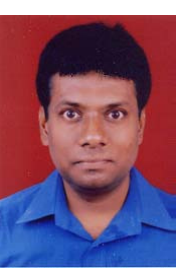
Instrumentation and Control Systems, International Journal of Control Systems and Computer Modelling, International Journal of Information Technology, Control and Automation, etc. His research interests are Linear and Nonlinear Control Systems, Chaos Theory and Control, Soft Computing, Optimal Control, Process Control, Operations Research, Mathematical Modelling, Scientific Computing using MATLAB etc. He has delivered several Key Note Lectures on Linear and Nonlinear Control Systems, Chaos Theory and Control, Scientific Computing using MATLAB/SCILAB, etc.

Mr. R. Karthikeyan obtained his M.Tech degree in Embedded Systems Technologies from Vinayaka Missions University, Tamil Nadu, India in 2007. He earned his B.E. degree in Electronics and Communication Engineering from Univeristy of Madras, Tamil $\mathrm{Nadu}$, in 2005. He has published over 10 papers in refereed International Journals. He has published several papers on Embedded Systems in National and International Conferences. His current research interests are Em bedded Systems, Robotics, Communications and Control Systems. 